\title{
Optimization of nonlinear structural resonance using the incremental harmonic balance method
}

\section{Dou, Suguang; Jensen, Jakob Søndergaard}

Published in:

Journal of Sound and Vibration

Link to article, DOI:

10.1016/j.jsv.2014.08.023

Publication date:

2015

Link back to DTU Orbit

Citation (APA):

Dou, S., \& Jensen, J. S. (2015). Optimization of nonlinear structural resonance using the incremental harmonic balance method. Journal of Sound and Vibration, 334, 239-254. https://doi.org/10.1016/j.jsv.2014.08.023

\section{General rights}

Copyright and moral rights for the publications made accessible in the public portal are retained by the authors and/or other copyright owners and it is a condition of accessing publications that users recognise and abide by the legal requirements associated with these rights.

- Users may download and print one copy of any publication from the public portal for the purpose of private study or research.

- You may not further distribute the material or use it for any profit-making activity or commercial gain

- You may freely distribute the URL identifying the publication in the public portal

If you believe that this document breaches copyright please contact us providing details, and we will remove access to the work immediately and investigate your claim 


\title{
Optimization of nonlinear structural resonance using the incremental harmonic balance method
}

\author{
Suguang Dou ${ }^{1}$, Jakob Søndergaard Jensen ${ }^{1,2}$ \\ 1 Department of Mechanical Engineering, Technical University of Denmark, 2800 Kgs. \\ Lyngby, Denmark, \\ 2 Department of Electrical Engineering, Technical University of Denmark, 2800 Kgs. \\ Lyngby, Denmark
}

\begin{abstract}
We present an optimization procedure for tailoring the nonlinear structural resonant response with time-harmonic loads. A nonlinear finite element method is used for modeling beam structures with a geometric nonlinearity and the incremental harmonic balance method is applied for accurate nonlinear vibration analysis. An optimization procedure based on a gradient-based algorithm is developed and we use the adjoint method for efficient computation of design sensitivities. We consider several examples in which we find optimized beam width distributions that minimize or maximize fundamental or super-harmonic resonant responses.
\end{abstract}

Keywords: optimization, nonlinear vibration, finite element, harmonic balance, adjoint method

\section{Introduction}

Nonlinear structural vibrations have drawn increasing attention in fields of energy harvesting devices and vibration-based microelectromechanical systems (MEMS). Rhoads et al. gave a comprehensive review of the application 
of nonlinear dynamics in Micro- and Nanoresonators concerning theoretical and experimental investigation of modeling, analysis and optimization [1]. A well known phenomenon in nonlinear vibration is the hardening and softening nonlinear effects $[2,3]$. Midplane stretching of a clamped-clamped beam, for example, results in a hardening nonlinearity that shifts the resonance frequency towards higher values for vibrations with higher amplitude. On one hand, this can undermine the performance of MEMS resonators serving as frequency references and demanding low acceleration sensitivity [4, 5]. On the other hand, it can be utilized to develop ultra-wide bandwith piezoelectric energy harvesting devices [6]. In both cases, incorporating nonlinear effects is an essential part in the design optimization of such structures.

For the computation of the steady-state response of nonlinear structural vibration, the principle of harmonic balance is widely used to develop efficient methods. Lau and Cheung proposed the incremental harmonic balance (IHB) method [7]. Chen et al. combined the IHB and finite element methods to analyze nonlinear vibrations of plane structures [8] whereas Lewandowski presented a general formulation for computing steady-state vibrations of geometrically nonlinear structures by using a harmonic balance and finite element method $[9,10,11]$. The classical IHB method is suitable for structural vibration with polynomial nonlinearity, whereas for structural vibration with a more complex nonlinearity, the alternating frequency/time (AFT) domain method proposed by Cameron [12] is widely used. The AFT method takes advantage of the convenience of evaluating nonlinear terms in the time domain. Maple et al. proposed an adaptive harmonic balance method for nonlinear time-periodic flows which results in a significant reduction in computational 
costs [13].

It is well known that harmonic balance methods can be combined with arc-length methods for path following $[14,15,16]$. Didier et al. summarized three predictors and three correctors for path following [17]. Beside the arc-length method, Leung and Fung proposed a phase increment method for path following of complicated frequency-amplitude curves [18, 19], and Cochelin and Vergez proposed an asymptotic numerical method (ANM) for continuation [20]. Hall et al. presented a high dimensional harmonic balance (HDHB) method $[21,22]$ where, instead of working in the frequency domain, the problem is cast into the time domain. Recently LaBryer and Attar presented a filtered HDHB method for large-scale problems in nonlinear structural dynamics [23] and Didier et al. proposed a stochastic multi-dimensional harmonic balance method (Stochastic-MHBM) for mechanical systems with non-regular nonlinearities and uncertainties [17]. The IHB method is adopted here because it is easy to implement for systems with quadratic and cubic nonlinearities and does not suffer from the aliasing problems that may occur using the AFT and HDHB methods.

In recent years, the principle of harmonic balance has been used on several occasions together with optimization procedures. Thothadri and Moon presented a nonlinear system identification method, which combined the principle of harmonic balance and bifurcation theory techniques to form a constrained optimization problem [24] and Ahmadian and Jalali used the incremental harmonic balance method and unconstrained optimization to identify nonlinear parameters for modeling bolted lap joints [25]. Coudeyras et al. proposed the constrained harmonic balance method together with finite ele- 
ment model to analyze the squeal of a brake system [26] whereas Liao and Sun used the principle of harmonic balance and the Hill method to form a constrained maximization problem for predicting the maximum vibration amplitude [27]. Here we apply the incremental harmonic balance method in conjunction with a shape optimization procedure to design geometrically nonlinear beam structures.

Shape optimization of beam structures has been considered by many researchers. Olhoff studied shape optimization of a thin, elastic beam with geometrically similar cross sections for maximizing higher order natural frequencies [28]. Olhoff and Parbery investigated shape optimization of vibrating beams and rotating shafts for maximizing the gap between two adjacent natural frequencies [29] and Bendsøe and Olhoff considered shape optimization of beams and rotating shafts for maximizing the distance from a given external excitation frequency or service speed to the nearest natural frequency in order to avoid resonance or whirling instability [30]. Meske et al. presented a new shape optimization method for natural frequency problems based on an optimality criterion, which is implemented in the optimization system TOSCA [31]. Olhoff et al. studied shape optimization of Bernoulli-Euler beams for maximizing the frequency gaps by using finite element method and gradient based optimization, and highlighted the associated periodicity and bandgaps of the optimized beam designs [32]. In the past years, shape optimization has also been a growing topic in the field of energy harvesting. Dietl and Garcia used optimization techniques to find the optimized shape of a beam with non-uniform width to improve the power output [33]. Wickenheiser demonstrated that beams with variable thickness lead to in- 
creased harvesting performance [34]. However, shape optimization of beam structures based on nonlinear vibrations with the focus on the nonlinear resonant response has to the authors' knowledge not been considered previously. In related work, Stanford et al. investigated shape optimization of a planar elastic rotating beam with geometric nonlinearities and time-periodic load by use of time marching method, spectral element method and finite difference method with/without model reduction based on proper orthogonal decomposition (POD) modes [35]. In further work, Stanford et al. optimized the stability and the peak power of nonlinear time-periodic flight and structural dynamics of flapping wing structures, where the wing is modeled as a geometrically nonlinear beam structure [36].

In the present paper we focus on developing an accurate and efficient method for optimization of nonlinear structural resonance with time-harmonic loads based on finite element analysis and the incremental harmonic balance method. We apply the proposed methodology to optimize several featured problems in nonlinear structural vibrations. Firstly, we formulate an optimization problem for the minimum amplitude of nonlinear resonant vibrations valid in the case of synchronous forcing. Secondly, we demonstrate the effectiveness of the methodology in minimizing/maximizing the amplitude of super-harmonic resonances. For all problems, the sensitivities are efficiently computed by the adjoint method and the iterative updates of design variables are found with a gradient-based strategy using a mathematical programming tool [37]. 


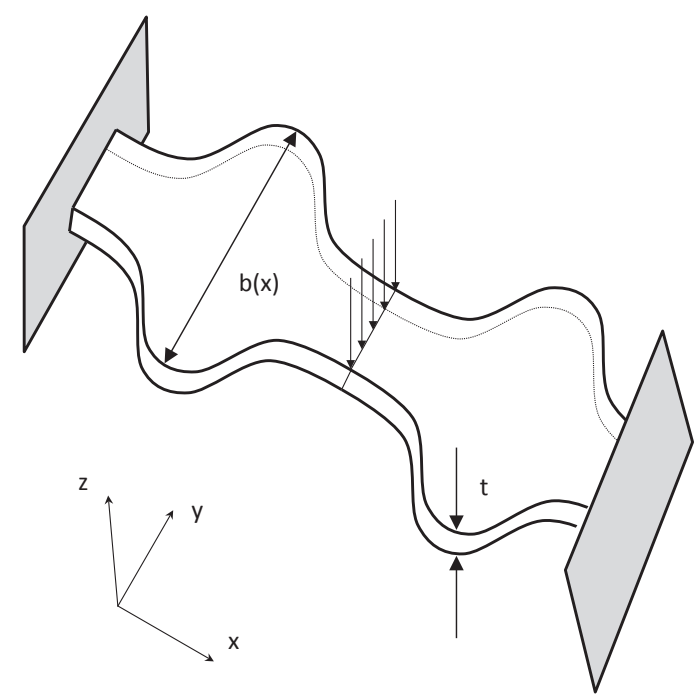

Figure 1: Schematic diagram of a doubly clamped beam with constant thickness and non-uniform width.

\section{Incremental harmonic balance method}

We consider the nonlinear structural vibrations of beam structures. The beam is assumed to have a constant thickness $t$ but a variable width $b(x)$ which distribution we aim to optimize in order to tailor the nonlinear structural response. A schematic of the optimization problem is shown in Figure 1.

\subsection{Nonlinear finite element method}

We begin with a geometrically non-linear beam element with quadratic and cubic nonlinearity arising from midplane stretching [8]. Since our primary interest is to demonstrate the methodology for optimization, large deformations and rotation are not taken into account here. The displacement 
vector is defined as

$$
\mathbf{u}(x, t)=\left[\begin{array}{ll}
u(x, t) & w(x, t)
\end{array}\right]^{T}
$$

where $u(x, t)$ and $w(x, t)$ is the axial and transverse displacement, respectively, and the axial strain $\epsilon_{0}$ and curvature $\kappa$ are defined as

$$
\epsilon_{0}=\frac{\partial u}{\partial x}+\frac{1}{2}\left(\frac{\partial w}{\partial x}\right)^{2}, \kappa=\frac{\partial^{2} w}{\partial x^{2}}
$$

where the quadratic term in the axial strain represents the effect of the midplane stretching. The nonlinear strain-displacement relation can be expressed as

$$
\epsilon=\epsilon_{0}+z \kappa
$$

where $z$ is the distance of the material particle in the cross section from the midplane. Assume the stress-strain relation is still linear as

$$
\sigma=E \epsilon
$$

where $E$ is Young's modulus.

Using equations (1)-(4) and ignoring rotational inertia, we obtain the kinetic energy $T$, the potential energy $U$ and the work $W$ done by external force and damping force as

$$
\begin{aligned}
T & =\sum_{e=1}^{N_{e}} \frac{1}{2} \int_{l_{e}} \rho A\left(\dot{u}^{2}+\dot{w}^{2}\right) d x \\
U & =\sum_{e=1}^{N_{e}} \frac{1}{2} \int_{l_{e}} E A \epsilon_{0}^{2}+E I \kappa^{2} d x \\
W & =\sum_{e=1}^{N_{e}} \int_{l_{e}} \mathbf{u}^{T} \mathbf{p}-\mu A \mathbf{u}^{T} \dot{\mathbf{u}} d x
\end{aligned}
$$


where the external force vector is expressed as

$$
\mathbf{p}=\left[\begin{array}{ll}
f_{u}(x, t) & f_{w}(x, t)
\end{array}\right]^{T}
$$

and $A$ is the cross-sectional area, $I$ the area moment of inertia, $\rho$ the mass density and $\mu$ is a viscous damping coefficient.

We now discretize the beam with a Galerkin finite element procedure by introducing the nodal displacement vectors $\mathbf{u}_{e}, \mathbf{w}_{e}$ and $\mathbf{q}_{e}$ associated with the finite element discretization as

$$
u=\mathbf{N}^{p} \mathbf{u}_{e}, w=\mathbf{N}^{b} \mathbf{w}_{e}, \mathbf{u}=\mathbf{N q}_{e}
$$

where

$$
\begin{aligned}
& \mathbf{u}_{e}=\left[\begin{array}{ll}
u_{i} & u_{j}
\end{array}\right]^{T}, \mathbf{w}_{e}=\left[\begin{array}{llll}
w_{i} & \theta_{i} & w_{j} & \theta_{j}
\end{array}\right]^{T}, \mathbf{q}_{e}=\left[\begin{array}{ll}
\mathbf{u}_{e}^{T} & \mathbf{w}_{e}^{T}
\end{array}\right]^{T} \\
& \mathbf{N}^{p}=\left[\begin{array}{ll}
N_{1} & N_{2}
\end{array}\right], \mathbf{N}^{b}=\left[\begin{array}{llll}
N_{3} & N_{4} & N_{5} & N_{6}
\end{array}\right], \mathbf{N}=\left[\begin{array}{cc}
\mathbf{N}^{p} & \mathbf{0} \\
\mathbf{0} & \mathbf{N}^{b}
\end{array}\right]
\end{aligned}
$$

The shape functions are selected as

$$
\begin{aligned}
& N_{1}=1-\xi, N_{2}=\xi, \xi=x / l_{e} \\
& N_{3}=1-3 \xi^{2}+2 \xi^{3}, N_{4}=l_{e}\left(\xi-2 \xi^{2}+\xi^{3}\right) \\
& N_{5}=3 \xi^{2}-2 \xi^{3}, N_{6}=l_{e}\left(\xi^{3}-\xi^{2}\right)
\end{aligned}
$$

By substituting equation (7) into equation (5) and applying the Hamilton's principle

$$
\int_{0}^{t} \delta T-\delta U+\delta W d t=0
$$

we obtain the global equation of motion for the beam structure as

$$
\mathbf{M} \ddot{\mathbf{q}}+\mathbf{C} \dot{\mathbf{q}}+\mathbf{g}(\mathbf{q})=\mathbf{f}
$$


where global matrices, $\mathbf{M}$ and $\mathbf{C}$, and vectors, $\mathbf{g}$ and $\mathbf{f}$, are assembled in the usual finite element way from the local element quantities, $\mathbf{M}_{e}, \mathbf{C}_{e}, \mathbf{g}_{e}$ and $\mathbf{f}_{e}$. The element mass matrix $\mathbf{M}_{e}$ and the damping matrix $\mathbf{C}_{e}$ are the same as for the linear problem; the element vector $\mathbf{g}_{e}$ represents the nonlinear elastic force and the vector $\mathbf{f}_{e}$ denotes the external force. Their specific forms are given as

$$
\begin{aligned}
& \mathbf{M}_{e}=\rho A \int_{0}^{l_{e}} \mathbf{N}^{T} \mathbf{N} d x, \mathbf{C}_{e}=\mu A \int_{0}^{l_{e}} \mathbf{N}^{T} \mathbf{N} d x, \mathbf{f}_{e}=\int_{0}^{l_{e}} \mathbf{N}^{T} \mathbf{p} d x \\
& \mathbf{g}_{e}\left(\mathbf{q}_{e}\right)=\mathbf{K}_{0} \mathbf{q}_{e}+\mathbf{f}_{\mathrm{nl}}, \mathbf{K}_{0}=\left[\begin{array}{cc}
\mathbf{K}_{u} & \mathbf{0} \\
\mathbf{0} & \mathbf{K}_{w}
\end{array}\right], \mathbf{f}_{\mathrm{n} 1}=\left[\begin{array}{c}
\mathbf{f}_{u}\left(\mathbf{w}_{e}\right) \\
\mathbf{f}_{w}\left(\mathbf{u}_{e}, \mathbf{w}_{e}\right)
\end{array}\right] \\
& \mathbf{K}_{u}=E A \int_{0}^{l_{e}}\left(\mathbf{B}_{0}^{p}\right)^{T} \mathbf{B}_{0}^{p} d x, \mathbf{K}_{w}=E I \int_{0}^{l_{e}}\left(\mathbf{B}_{0}^{b}\right)^{T} \mathbf{B}_{0}^{b} d x \\
& \mathbf{f}_{u}=E A \int_{0}^{l_{e}} \frac{1}{2}\left(\mathbf{B}_{0}^{p}\right)^{T}\left(\mathbf{G w}_{e}\right)^{2} d x \\
& \mathbf{f}_{w}=E A \int_{0}^{l_{e}} \mathbf{G}^{T}\left(\mathbf{G w}_{e}\right)\left(\mathbf{B}_{0}^{p} \mathbf{u}_{e}\right)+\frac{1}{2} \mathbf{G}^{T}\left(\mathbf{G w}_{e}\right)^{3} d x \\
& \mathbf{B}_{0}^{p}=\frac{\partial \mathbf{N}^{p}}{\partial x}, \mathbf{B}_{0}^{b}=\frac{\partial^{2} \mathbf{N}^{b}}{\partial x^{2}}, \mathbf{G}=\frac{\partial \mathbf{N}^{b}}{\partial x}
\end{aligned}
$$

\subsection{Incremental harmonic balance method}

The derivations in the following can be found in similar form in reference [8]. Here, we have reorganized the derivations and included details for completeness in combination with the forthcoming sensitivity analysis.

First we introduce a non-dimensional time scale $\tau=\omega t$ and rewrite the equation of motion (11) as

$$
\omega^{2} \mathbf{M} \mathbf{q}^{\prime \prime}+\omega \mathbf{C q} \mathbf{q}^{\prime}+\mathbf{g}=\mathbf{f}
$$

where the prime $\left(^{\prime}\right)$ represents the differentiation with respect to $\tau$. 
The IHB method effectively combines a Newton-Raphson procedure with the harmonic balance approach. Most commonly the incremental procedure is applied before the Newton-Raphson procedure. However, in order to highlight the governing equation of the equilibrium state in the frequency domain, we apply the harmonic balance method first. The harmonic balance method starts with the assumption that the external loads and the dynamic response are periodic as

$$
\begin{aligned}
& f_{i}=f_{i 0}+\sum_{n=1}^{N_{H}}\left(f_{i n}^{c} \cos (n \tau)+f_{i n}^{s} \sin (n \tau)\right)=\mathbf{C}_{S} \mathcal{F}\left(f_{i}\right) \\
& q_{i}=a_{i 0}+\sum_{n=1}^{N_{H}}\left(a_{i n} \cos (n \tau)+b_{i n} \sin (n \tau)\right)=\mathbf{C}_{S} \mathcal{F}\left(q_{i}\right)
\end{aligned}
$$

where

$$
\mathbf{C}_{S}=\left[\begin{array}{lllllll}
1 & \cos \tau & \ldots & \cos \left(N_{H} \tau\right) & \sin \tau & \ldots & \sin \left(N_{H} \tau\right)
\end{array}\right]
$$

and $\mathcal{F}($ ) denotes the coefficient vector of the Fourier series projected on the basis $\mathbf{C}_{S}$. For convenience and conciseness, the global displacement vector $\mathbf{q}$ is expressed as

$$
\mathbf{q}=\mathbf{S} \overline{\mathbf{q}}
$$

where $\overline{\mathbf{q}}$ collects all the coefficients $a_{i n}$ and $b_{i n}$ in the Fourier expansion, and

$$
\mathbf{S}=\operatorname{diag}\left(\left[\begin{array}{lll}
\mathbf{C}_{S} & \ldots & \mathbf{C}_{S}
\end{array}\right]\right)
$$

The essence of the harmonic balance method is to eliminate the time dimension and reform the differential equation into a set of algebraic equations. Substituting equation (14) into equation (13), and applying the principle of harmonic balance, one can obtain a set of non-linear equations with $a_{\text {in }}$ and 
$b_{i n}$ as unknowns. A procedure in matrix format is obtained by substituting equations (16) into equation (13) and applying the Galerkin method using the left multiplication with $\mathbf{S}^{T}$ and integration from 0 to $2 \pi$. In this case, one can readily obtain a set of equations in matrix form as

$$
\omega^{2} \overline{\mathbf{M}} \overline{\mathbf{q}}+\omega \overline{\mathbf{C}} \overline{\mathbf{q}}+\overline{\mathbf{g}}=\overline{\mathbf{f}}
$$

This governing equation can be viewed as a frequency domain representation of the equation of motion in (11). The barred terms $\overline{\mathbf{M}}, \overline{\mathbf{C}}, \overline{\mathbf{g}}$ and $\overline{\mathbf{f}}$ are given as

$$
\begin{aligned}
& \overline{\mathbf{M}}=\frac{1}{2 \pi} \int_{0}^{2 \pi} \mathbf{S}^{T} \mathbf{M} \mathbf{S}^{\prime \prime} d \tau, \overline{\mathbf{g}}=\frac{1}{2 \pi} \int_{0}^{2 \pi} \mathbf{S}^{T} \mathbf{g} d \tau, \\
& \overline{\mathbf{C}}=\frac{1}{2 \pi} \int_{0}^{2 \pi} \mathbf{S}^{T} \mathbf{C} \mathbf{S}^{\prime} d \tau, \overline{\mathbf{f}}=\frac{1}{2 \pi} \int_{0}^{2 \pi} \mathbf{S}^{T} \mathbf{f} d \tau
\end{aligned}
$$

The second step of the incremental harmonic balance method is to obtain the incremental equation. Let $\mathbf{q}_{0}$ and $\omega_{0}$ denote a known solution in amplitude and frequency space. The state in the neighborhood of the known state can be expressed as

$$
\mathbf{q}=\mathbf{q}_{0}+\Delta \mathbf{q}, \quad \omega=\omega_{0}+\Delta \omega
$$

Inserting equation (20) into equation (18) and collecting the first-order incremental terms, we obtain

$$
\overline{\mathbf{K}}_{\overline{\mathbf{q}}} \Delta \overline{\mathbf{q}}+\overline{\mathbf{K}}_{\omega} \Delta \omega=\overline{\mathbf{r}}
$$

in which

$$
\begin{aligned}
& \overline{\mathbf{K}}_{\overline{\mathbf{q}}}=\omega_{0}^{2} \overline{\mathbf{M}}+\omega_{0} \overline{\mathbf{C}}+\overline{\mathbf{K}}_{T} \\
& \overline{\mathbf{K}}_{\omega}=2 \omega_{0} \overline{\mathbf{M}} \overline{\mathbf{q}}_{0}+\overline{\mathbf{C}} \overline{\mathbf{q}}_{0} \\
& \overline{\mathbf{r}}=\overline{\mathbf{f}}-\omega_{0}^{2} \overline{\mathbf{M}} \overline{\mathbf{q}}_{0}-\omega_{0} \overline{\mathbf{C}} \overline{\mathbf{q}}_{0}-\overline{\mathbf{g}}
\end{aligned}
$$


The barred terms $\overline{\mathbf{K}}_{T}$ are obtained by integration from 0 to $2 \pi$ and recalling equation (16) as

$$
\overline{\mathbf{K}}_{T}=\frac{1}{2 \pi} \int_{0}^{2 \pi} \mathbf{S}^{T} \frac{\partial \mathbf{g}}{\partial \mathbf{q}} \frac{\partial \mathbf{q}}{\partial \overline{\mathbf{q}}} d \tau=\frac{1}{2 \pi} \int_{0}^{2 \pi} \mathbf{S}^{T} \mathbf{K}_{T} \mathbf{S} d \tau
$$

It is noted that the matrix $\mathbf{K}_{T}$ represents the tangent stiffness matrix, and $\overline{\mathbf{K}}_{T}$ denotes its counterpart in the frequency domain.

\subsection{Numerical implementation}

In the finite element formulation, the shape functions for the stretching and bending contributions are not consistent for $\epsilon_{0}$ in equation (2), and this causes a locking problem and an unphysical hardening nonlinearity. To overcome this problem, an average approximation of the axial strain is used [8]. Introduce a constant matrix as

$$
\mathbf{K}_{g}=\int_{0}^{l_{e}} \mathbf{G}^{T} \mathbf{G} d x
$$

where $\mathbf{G}$ is defined in equation (12). Then the average value of the axial strain in one beam element is constant as

$$
\epsilon_{0}=\mathbf{B}_{0}^{p} \mathbf{u}_{e}+\frac{1}{2 l_{e}} \mathbf{w}_{e}^{T} \mathbf{K}_{g} \mathbf{w}_{e}
$$

The nonlinear internal forces in equation (12) are re-expressed as

$$
\begin{aligned}
& \mathbf{f}_{u}=\frac{1}{2} E A\left(\mathbf{B}_{0}^{p}\right)^{T} \mathbf{w}_{e}^{T} \mathbf{K}_{g} \mathbf{w}_{e} \\
& \mathbf{f}_{w}=E A\left(\mathbf{B}_{0}^{p} \mathbf{u}_{e}+\frac{1}{2 l_{e}} \mathbf{w}_{e}^{T} \mathbf{K}_{g} \mathbf{w}_{e}\right) \mathbf{K}_{g} \mathbf{w}_{e}=T_{0} \mathbf{K}_{g} \mathbf{w}_{e}
\end{aligned}
$$

where $T_{0}$ is the average value of axial force. The specific form of the tangent stiffness matrix $\mathbf{K}_{T}$ is written in three parts as

$$
\mathbf{K}_{T}=\mathbf{K}_{0}+\mathbf{K}_{L}+\mathbf{K}_{\sigma}
$$


where

$$
\begin{aligned}
& \mathbf{K}_{L}=\left[\begin{array}{ll}
\mathbf{0} & \mathbf{K}_{u w} \\
\mathbf{K}_{w u} & \mathbf{K}_{w w}
\end{array}\right], \mathbf{K}_{\sigma}=\left[\begin{array}{ll}
\mathbf{0} & \mathbf{0} \\
\mathbf{0} & \mathbf{K}_{\sigma}^{b}
\end{array}\right] \\
& \mathbf{K}_{u w}=E A\left(\mathbf{B}_{0}^{p}\right)^{T} \mathbf{w}_{e}^{T} \mathbf{K}_{g}, \mathbf{K}_{w u}=\mathbf{K}_{u w}^{T} \\
& \mathbf{K}_{w w}=\frac{E A}{l_{e}} \mathbf{K}_{g} \mathbf{w}_{e} \mathbf{w}_{e}^{T} \mathbf{K}_{g} \\
& \mathbf{K}_{\sigma}^{b}=E A\left(\mathbf{B}_{0}^{p} \mathbf{u}_{e}+\frac{1}{2 l_{e}} \mathbf{w}_{e}^{T} \mathbf{K}_{g} \mathbf{w}_{e}\right) \mathbf{K}_{g}=T_{0} \mathbf{K}_{g}
\end{aligned}
$$

Note that $\mathbf{K}_{T}$ is symmetric. Symbolic calculations are done for equations (26) and (28), and the coefficients of quadratic and cubic terms are automatically extracted.

To perform the integration in equations (19) and (23) it can be exploited that following matrices can be analytically precomputed:

$$
\begin{aligned}
& \mathbf{H}^{(0)}=\frac{1}{2 \pi} \int_{0}^{2 \pi} \mathbf{C}_{S}^{T} \mathbf{C}_{S}^{\prime \prime} d \tau=\frac{1}{2} \operatorname{diag}\left(\left[0-1 \cdots-N_{H}^{2}-1 \cdots\right.\right. \\
& \mathbf{H}^{(1)}=\frac{1}{2 \pi} \int_{0}^{2 \pi} \mathbf{C}_{S}^{T} \mathbf{C}_{S}^{\prime} d \tau=\left[\begin{array}{ccc}
0 & \mathbf{0} & \mathbf{0} \\
\mathbf{0} & \mathbf{0} & \mathbf{c} \\
\mathbf{0} & -\mathbf{c} & \mathbf{0}
\end{array}\right], \mathbf{c}=\frac{1}{2} \operatorname{diag}\left(\left[\begin{array}{lll}
1 & \cdots & N_{H}
\end{array}\right]\right) \\
& \mathbf{H}^{(2)}=\frac{1}{2 \pi} \int_{0}^{2 \pi} \mathbf{C}_{S}^{T} \mathbf{C}_{S} d \tau=\frac{1}{2} \operatorname{diag}\left(\left[\begin{array}{lllllll}
2 & 1 & \cdots & 1 & 1 & \cdots & 1
\end{array}\right]\right) \\
& \mathbf{H}^{(3)}(m, n, k)=\frac{1}{2 \pi} \int_{0}^{2 \pi} \mathbf{C}_{S}(m) \mathbf{C}_{S}(n) \mathbf{C}_{S}(k) d \tau, \\
& m, n, k=1,2, \ldots, 2 N_{H}+1
\end{aligned}
$$


with the components of $\mathbf{H}^{(3)}$ expressed as

$$
\begin{aligned}
& \mathbf{H}^{(3)}(1, m, n)=\mathbf{H}^{(3)}(m, 1, n)=\mathbf{H}^{(3)}(m, n, 1)=\mathbf{H}^{(2)}(m, n), \\
& c c c=\frac{1}{4}[\delta(i-j+k)+\delta(i-j-k)+\delta(i+j+k)+\delta(i+j-k)], \\
& s s c=\frac{1}{4}[\delta(i-j+k)+\delta(i-j-k)-\delta(i+j+k)-\delta(i+j-k)], \\
& \mathbf{H}^{(3)}\left(i_{s}, j_{s}, k_{c}\right)=\mathbf{H}^{(3)}\left(i_{s}, k_{c}, j_{s}\right)=\mathbf{H}^{(3)}\left(k_{c}, i_{s}, j_{s}\right)=s s c, \\
& \mathbf{H}^{(3)}\left(i_{c}, j_{c}, k_{c}\right)=c c c, \quad i, j, k=1, \ldots, N_{H}, \quad m, n=1, \ldots, 2 N_{H}+1, \\
& i_{c}=i+1, \quad j_{c}=j+1, \quad k_{c}=k+1, \quad i_{s}=i_{c}+N_{H}, \quad j_{s}=j_{c}+N_{H}, \\
& \delta(n)=1, \quad \text { if } n=0 ; \quad \delta(n)=0, \text { if } n \neq 0 .
\end{aligned}
$$

which saves considerable computational time when performing the iterative analysis. The barred terms in the equations (19) and (23) are calculated by using the precomputed matrices as

$$
\begin{array}{r}
\mathbf{M}(i, j) \longrightarrow \mathbf{M}(i, j) \mathbf{H}^{(0)}, \mathbf{C}(i, j) \longrightarrow \mathbf{C}(i, j) \mathbf{H}^{(1)} \\
\mathbf{g}(i) \longrightarrow \mathbf{H}^{(2)} \mathcal{F}(\mathbf{g}(i)), \mathbf{f}(i) \longrightarrow \mathbf{H}^{(2)} \mathcal{F}(\mathbf{f}(i)) \\
\mathbf{K}_{T}(\mathbf{q})(i, j) \longrightarrow \mathbf{H}^{(3)} \odot \mathcal{F}\left(\mathbf{K}_{T}(\mathbf{q})(i, j)\right)
\end{array}
$$

where the arrow " $\longrightarrow$ " represents a map from one entry in the matrix to one block in the augmented matrix, and " $\odot$ " represents the dot multiplication of the third dimension of the $\mathbf{H}^{(3)}$ with the vector $\mathcal{F}\left(\mathbf{K}_{T}(\mathbf{q})(i, j)\right)$, and yields a two-dimensional matrix.

As for the problem size, the total number of unknown variables equals the product of $N_{\mathrm{DOF}}$, the degrees of freedom of the finite element model, and $\left(2 N_{H}+1\right)$, the number of harmonic terms used for each degree of freedom. If the finite element matrices have a band structure, the augmented matrices are sparse matrices with band structure and the bandwidth is $\left(2 N_{H}+1\right)$ times 
that of the finite element matrices. Since only a few iterations are required to find the dynamic equilibrium, it is faster than the time marching method.

\subsection{Numerical solution techniques}

We adapt the incremental equation (21) in several ways to solve equation (18). Of primary interest is the frequency-response curve which is reflected in the choice of specifying the increments in the frequency-amplitude space in equation (20). For very weakly nonlinear problems and non-resonant states we can apply a simple frequency-increment scheme where $\Delta \omega$ is specified and equation (21) is solved for amplitude increments $\Delta \overline{\mathbf{q}}$. However for general path following of the frequency-amplitude curve this technique is not sufficient.

\subsubsection{The arc-length method}

Here, the arc-length of the frequency-amplitude curve is increased incrementally and terminated when the solution is outside a prescribed region. In equation (21), both the frequency increment and amplitude increments are treated as unknowns $\Delta \mathbf{X}=\left[\Delta \overline{\mathbf{q}}^{T} \Delta \omega\right]^{T}$, and a constraint is imposed so as to determine the solution. The arc-length constraint employed is

$$
\beta\left(\frac{\omega-\omega_{0}}{\omega_{0}}\right)^{2}+\left(\frac{\left\|\overline{\mathbf{q}}-\overline{\mathbf{q}}_{0}\right\|}{\left\|\overline{\mathbf{q}}_{0}\right\|}\right)^{2}=(\Delta s)^{2}
$$

where $\beta$ is 1 in the current algorithm. This can be viewed a corrector step. In the predictor step, the cubic extrapolation is used [14]. It should be noted that the predictor can also use the normalization procedure in equation (32). Step control is very important for developing a robust and efficient method. The step size is decided according to the ratio between the desirable number 
$N^{*}$ and the previous number $N_{k-1}$ of iterations [38] so as $s_{k+1}=N^{*} / N_{k} \times s_{k}$. In practice, the ratio $r=N^{*} / N_{k}$ is bounded to achieve smooth change of step size [39]. For small-scale equations, $N^{*}=3$ is a desirable value. For large-scale equations, $N^{*}$ should be larger. A maximum number of iterations $N_{\max }$ is used to make the code robust and efficient. However, the iteration may fail to converge, if the matrix becomes singular and the residual then increases drastically. Besides, the solution may jump to another branch, which can be identified through the length of real step size and the angle between consecutive predictor steps. In cases of no convergence and jumping, the step size is halved until convergence is achieved. The convergence criteria for the arc-length method are $\|\Delta \mathbf{X}\|<\epsilon_{\mathbf{X}}\left\|\Delta \mathbf{X}_{0}\right\|$ and $\|\overline{\mathbf{r}}\|<\epsilon_{\overline{\mathbf{r}}}\|\overline{\mathbf{f}}\|$.

\subsubsection{Resonance peak analysis}

In order to optimize the peak of primary resonance, a simple criterion is required to identify the peak. In previous work, Petrov defined the resonance peak as the local maximum point with $\frac{\partial a}{\partial \omega}=0$, where $a=\frac{1}{2} \sqrt{a_{i n}^{2}+b_{i n}^{2}}, i$ and $n$ denotes the $i^{\text {th }}$ degree of freedom and $n^{\text {th }}$ harmonic, respectively [40]. Likewise, the folding point with $\frac{\partial \omega}{\partial a}=0$ may also be tracked and optimized. In both schemes, second-order sensitivity analysis is required in optimization. Here in the case of synchronous forcing, we track the resonance peak by using the point whose displacement response has $90^{\circ}$ phase lag relative to the harmonic loads, which only requires the first-order sensitivity analysis in optimization. So in addition to the arc-length method we also apply a specialized scheme for resonance peak analysis. For this scheme we increase the external load $\overline{\mathbf{f}}$ in incremental steps with one Fourier coefficient $\overline{\mathbf{q}}(i)$ and its corresponding increment $\Delta \overline{\mathbf{q}}(i)$ constantly fixed to zero in equation (21). 
The load starts from a small value and the initial guess is obtained using least square method or Moore-Penrose pseudo-inverse method with $\omega=\omega_{i}$ and $\Delta \omega=0$. For each load increment, the corresponding frequency increment $\Delta \omega$ and the other Fourier coefficient increments $\Delta \overline{\mathbf{q}}(j)$ with $j \neq i$ are then solved iteratively. This provides an efficient way to evaluate the peak of the primary resonance. This scheme provides an efficient way to evaluate the peak of the primary resonance and will be further outlined in the following section.

\subsection{Nonlinear vibrations of a uniform beam}

In the following a beam with uniform width will be analyzed. This will also be the reference beam used in the optimization procedure. The parameters of this beam are: $b=30 \mathrm{~mm}, h=10 \mathrm{~mm}, E=2.05 \times 10^{5} \mathrm{MPa}, \rho=$ $7.8 \times 10^{-9}$ tonne $/ \mathrm{mm}^{3}, A=b h, I=\left(b h^{3}\right) / 12, r=\sqrt{I / A}, L=150 r$. The damping model is introduced in equation (5) and leads to a damping matrix proportional to the mass matrix, i.e. $\mathbf{C}=\alpha \mathbf{M}$, with $\alpha=\frac{\mu}{\rho}$. Assuming $\alpha=2 \xi \omega_{1}$, then the damping coefficient $\mu$ is given by $\mu=2 \rho \xi \omega_{1}$, where $\omega_{1}$ is the first eigenvalue of the initial design of a doubly clamped beam and the damping ratio $\xi$ is chosen as 0.004 . Note that $r$ is the radius of gyration of the cross section area and the amplitudes in the following results are scaled with $1 / r$. Two load cases are considered including weak excitation with $f=15 E \operatorname{Ir} / L^{3}$ and a higher excitation level with $f=200 E \operatorname{Ir} / L^{3}$. The location and direction of the excitation force are shown in Figure (1).

Here the beam is discretized with 100 elements and up to sixth-order harmonics are used in the Fourier expansion. The primary resonance of this beam with doubly clamped boundary condition is shown in Figure 2. Weak 
excitation with $f=15 E \operatorname{Ir} / L^{3}$ is used and the frequency-amplitude curve is computed using frequency increments as initial steps and the arc-length method as the path-following steps. As seen in the figure, the nonlinear response includes a shift of the resonance frequency, folding of the response curve, jumping phenomena and multiple solutions. The peak of the resonance shifts towards higher frequencies, which represents hardening nonlinearity. In certain frequency ranges there are three solutions for one specific frequency. The top and bottom branches are stable, and the middle branch is unstable. So when the frequency sweeps upwards, the solution jumps to the bottom branch at the peak of the resonance. When the frequency sweeps downwards, the solution jumps to the top branch at the folding point. These are all well known phenomena but emphasize the need for an efficient and robust method to predict and subsequently optimize the resonance peak in nonlinear vibration.

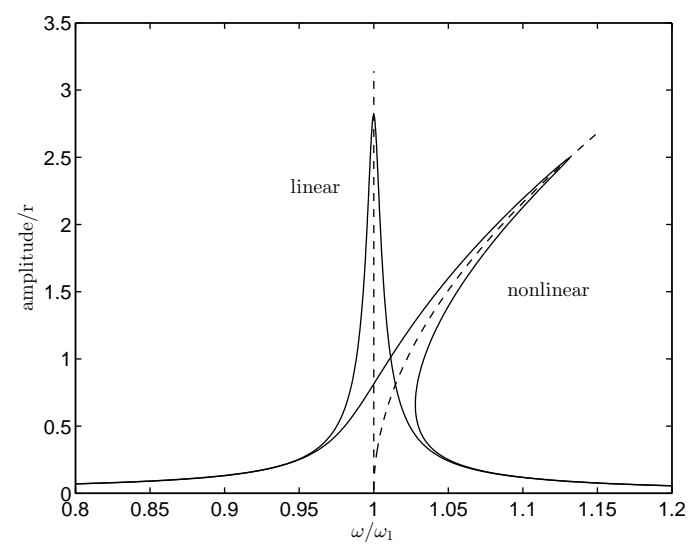

Figure 2: Forced vibration of a doubly clamped beam around the first natural frequency $\omega_{1}$. Solid lines denote the frequency-amplitude curves for forced vibration. Dashed lines denote the backbone computed with the phase lag quadrature criterion. 
In the following we outline a simple and efficient method to predict the peak of the resonance based on the backbone of the primary resonance shown in Figure 2. The backbone is obtained by path following of the resonant state with a phase lag of 90 degrees in the fundamental harmonic relative to the external excitation. This idea originates in the phase-lag quadrature criterion: for a structure with well separated modes, when it vibrates close to the peak of primary resonance, the periodic response of displacement crosses a phase lag of 90 degrees with respect to the excitation. Peeters and Kerschen et al. have theoretically and experimentally demonstrated that the phase-lag quadrature criterion used in linear vibration can be generalized to nonlinear vibrations $[41,42]$. A physical interpretation is that the external load compensates for the damping force. For nonlinear vibrations, the phaselag criterion can be defined for each harmonic and when the periodic load is written as a sine series

$$
f_{i}=\sum_{n=1}^{N_{H}} f_{i n}^{s} \sin (n \tau)
$$

the nonlinear response fulfilling the phase lag quadrature criterion is

$$
q_{i}=\sum_{n=1}^{N_{H}} a_{i n} \cos (n \tau)
$$

In this case, all the coefficients $b_{i n}$ of sine terms become zero. In our analysis, only $b_{i 1}$ of the fundamental harmonic is zero, i.e.

$$
b_{i 1}=0
$$

and the increments of the other Fourier coefficients and the frequency are found by the incremental scheme. Note, that the assumption that the phase 
lag between displacements and excitation is $90^{\circ}$ at the resonance is valid only in the case when all forces are synchronous.

The superharmonic resonance of this beam with doubly clamped boundary conditions is shown in Figure 3 with higher excitation level $\left(f=200 E \operatorname{Ir} / L^{3}\right)$ used to induce the superharmonic resonance. For linear vibrations, there is only one fundamental harmonic whereas in the non-linear case there are multiple harmonics. It is seen clearly that the amplitude of the third harmonic is resonantly excited and we refer to this as a superharmonic resonance. In the following part, we also propose an optimization scheme to optimize the amplitude of the superharmonic resonance.

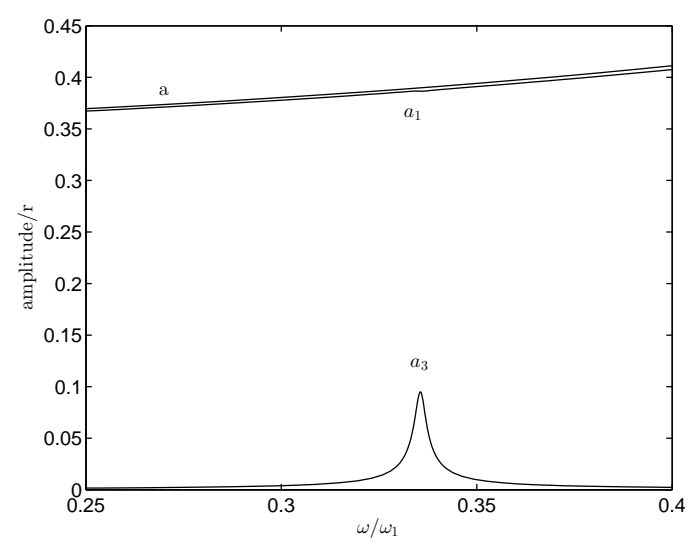

Figure 3: Superharmonic resonance of a doubly clamped beam near $\omega_{1} / 3$. a denotes the amplitude of the fundamental harmonic in linear vibration. $a_{1}$ and $a_{3}$ denote the amplitudes of the first-order and third-order harmonics in nonlinear vibration.

\section{Optimization problem}

Our optimization problem is to minimize (or alternatively maximize) the response amplitude for primary and superharmonic resonances by optimizing 
the width distribution of the beam. The optimization problems for primary and superharmonic resonances are formulated in slightly different ways as follows.

\subsection{Optimization of primary resonance}

Based on the phase-lag quadrature criterion and by assuming that the external excitation is a pure sine function, a specific formulation for minimizing the amplitude of the peak of primary resonance is written as

$$
\begin{array}{ll}
\min _{\rho_{e}} & c=\overline{\mathbf{q}}^{T} \mathbf{L} \overline{\mathbf{q}} \\
\text { s.t. }: & b_{i 1}=0, \\
& \omega^{2} \overline{\mathbf{M}} \overline{\mathbf{q}}+\omega \overline{\mathbf{C}} \overline{\mathbf{q}}+\overline{\mathbf{g}}=\overline{\mathbf{f}}, \\
& \sum_{e=1}^{N_{e}} b_{e} \leq \alpha N_{e} b_{\max }, \\
& b_{e}=b_{\min }+\rho_{e}\left(b_{\max }-b_{\min }\right), \\
& 0 \leq \rho_{e} \leq 1 .
\end{array}
$$

where $\mathbf{L}$ is a diagonal matrix with unit entries that define which components of $\overline{\mathbf{q}}$ that are considered, $\rho_{e}\left(e=1, \ldots, N_{e}\right)$ are the design variables that are used to control the width of each beam element $b_{e}$, and $\alpha$ defines a volume constraint by specifying a maximum allowable design volume fraction of the total admissible design domain $N_{e} b_{\max }$ (where the constant beam thickness $t$ has been omitted in the formulas). As we will explain in the subsequent examples it makes sense to specify a minimum volume for some optimization problems.

With $\omega$ determined from the phase-lag quadrature criterion we need to modify the general equations slightly. We write governing equation of the 
peak of the primary resonance as

$$
\omega^{2} \overline{\overline{\mathbf{M}}} \overline{\overline{\mathbf{q}}}+\omega \overline{\overline{\mathbf{C}}} \overline{\overline{\mathbf{q}}}+\overline{\mathbf{g}}=\overline{\mathbf{f}}-\left(\omega^{2} \overline{\mathbf{M}}_{j} \overline{\mathbf{q}}_{j}+\omega \overline{\mathbf{C}}_{j} \overline{\mathbf{q}}_{j}\right)
$$

where $\overline{\overline{\mathbf{M}}}$ is the same as $\overline{\mathbf{M}}$ except the $j^{\text {th }}$ column $\overline{\mathbf{M}}_{j}$ is taken out, $\overline{\overline{\mathbf{C}}}$ is the same as $\overline{\mathbf{C}}$ except the $j^{\text {th }}$ column $\overline{\mathbf{C}}_{j}$ is taken out, $\overline{\overline{\mathbf{q}}}$ is the same as $\overline{\mathbf{q}}$ except the $j^{\text {th }}$ component $\overline{\mathbf{q}}_{j}$ is taken out, and $\overline{\mathbf{q}}_{j}=b_{i 1}=0$. The incremental form used in the computation is

$$
\begin{aligned}
& \left(\omega^{2} \overline{\overline{\mathbf{M}}}+\omega \overline{\overline{\mathbf{C}}}+\overline{\overline{\mathbf{K}}}_{T}\right) \Delta \overline{\overline{\mathbf{q}}}+(2 \omega \overline{\mathbf{M}} \overline{\mathbf{q}}+\overline{\mathbf{C}} \overline{\mathbf{q}}) \Delta \omega \\
& =\overline{\mathbf{f}}-\left(\omega^{2} \overline{\mathbf{M}} \overline{\mathbf{q}}+\omega \overline{\mathbf{C}} \overline{\mathbf{q}}+\overline{\mathbf{g}}\right)
\end{aligned}
$$

where $\overline{\mathbf{K}}_{T}$ is the same as $\overline{\mathbf{K}}_{T}$ except the $j^{\text {th }}$ column is taken out. The sensitivity of $\overline{\overline{\mathbf{q}}}$ with respect to $\rho_{e}$ can be found through

$$
\begin{aligned}
& {\left[\begin{array}{cc}
\omega^{2} \overline{\mathbf{M}}+\omega \overline{\overline{\mathbf{C}}}+\overline{\overline{\mathbf{K}}}_{T} & 2 \omega \overline{\mathbf{M}} \overline{\mathbf{q}}+\overline{\mathbf{C}} \overline{\mathbf{q}}
\end{array}\right]\left[\begin{array}{c}
\frac{\mathrm{d} \overline{\mathbf{q}}}{\mathrm{d} \rho_{e}} \\
\frac{\mathrm{d} \omega}{\mathrm{d} \rho_{e}}
\end{array}\right]} \\
& =-\left(\omega^{2} \frac{\partial \overline{\mathbf{M}}}{\partial \rho_{e}} \overline{\mathbf{q}}+\omega \frac{\partial \overline{\mathbf{C}}}{\partial \rho_{e}} \overline{\mathbf{q}}+\frac{\partial \overline{\mathbf{g}}}{\partial \rho_{e}}\right)
\end{aligned}
$$

and the sensitivity of $c$ with respect to $\rho_{e}$ can then be expressed as

$$
\frac{\mathrm{d} c}{\mathrm{~d} \rho_{e}}=2 \boldsymbol{\lambda}^{T}\left(\omega^{2} \frac{\partial \overline{\mathbf{M}}}{\partial \rho_{e}} \overline{\mathbf{q}}+\omega \frac{\partial \overline{\mathbf{C}}}{\partial \rho_{e}} \overline{\mathbf{q}}+\frac{\partial \overline{\mathbf{g}}}{\partial \rho_{e}}\right)
$$

with the following adjoint equation

$$
\left[\omega^{2} \overline{\overline{\mathbf{M}}}+\omega \overline{\overline{\mathbf{C}}}+\overline{\overline{\mathbf{K}}}_{T} \quad 2 \omega \overline{\mathbf{M}} \overline{\mathbf{q}}+\overline{\mathbf{C}} \overline{\mathbf{q}}\right]^{T} \boldsymbol{\lambda}=-\mathbf{L}_{1}\left[\overline{\overline{\mathbf{q}}}^{T} \omega\right]^{T}
$$

where $\mathbf{L}_{1}$ is defined so as $\overline{\mathbf{q}}^{T} \mathbf{L} \overline{\mathbf{q}}=\left[\overline{\overline{\mathbf{q}}}^{T} \omega\right] \mathbf{L}_{1}\left[\overline{\overline{\mathbf{q}}}^{T} \omega\right]^{T}$.

\subsection{Optimization of superharmonic resonance}


In optimization of superharmonic resonances, we find it is effective to optimize the resonance peak by controlling the responses at $\omega=\gamma \omega_{i}$, where $\omega_{i}$ is the $i^{\text {th }}$ eigenvalue, $\gamma$ is a selected constant value that corresponds to superharmonic resonances. The optimization problem is now formulated as

$$
\begin{aligned}
& \min _{\rho_{e}} c=\overline{\mathbf{q}}^{T} \mathbf{L} \overline{\mathbf{q}} \\
\text { s.t. }: & \omega=\gamma \omega_{i}
\end{aligned}
$$

subjected also to the additional constraints from equation (36) (except $b_{i 1}=$ $0)$.

The sensitivity of the objective function w.r.t. our design variables can be derived through direct differentiation or an adjoint approach using Lagrange multipliers. For an efficient implementation with a large number of design variables we adhere to the latter approach and rewrite the objective function with a Lagrange multiplier as

$$
c=\overline{\mathbf{q}}^{T} \mathbf{L} \overline{\mathbf{q}}+2 \boldsymbol{\lambda}^{T}\left(\omega^{2} \overline{\mathbf{M}} \overline{\mathbf{q}}+\omega \overline{\mathbf{C}} \overline{\mathbf{q}}+\overline{\mathbf{g}}\right)
$$

Note that the Lagrange multiplier can take an arbitrary value. The sensitivity of $c$ with respect to $\rho_{e}$ can now be written as

$$
\frac{\mathrm{d} c}{\mathrm{~d} \rho_{e}}=2 \overline{\mathbf{q}}^{T} \mathbf{L} \frac{\mathrm{d} \overline{\mathbf{q}}}{\mathrm{d} \rho_{e}}+2 \boldsymbol{\lambda}^{T}\left[\begin{array}{c}
\omega^{2} \frac{\partial \overline{\mathbf{M}}}{\partial \rho_{e}} \overline{\mathbf{q}}+\omega \frac{\partial \overline{\mathbf{C}}}{\partial \rho_{e}} \overline{\mathbf{q}}+\frac{\partial \overline{\mathbf{g}}}{\partial \rho_{e}}+ \\
\left(\omega^{2} \overline{\mathbf{M}}+\omega \overline{\mathbf{C}}+\overline{\mathbf{K}}_{T}\right) \frac{\mathrm{d} \overline{\mathbf{q}}}{\mathrm{d} \rho_{e}}+ \\
(2 \omega \overline{\mathbf{M}} \overline{\mathbf{q}}+\overline{\mathbf{C}} \overline{\mathbf{q}}) \frac{\mathrm{d} \omega}{\mathrm{d} \rho_{e}}
\end{array}\right]
$$

where it should be noted that we include the excitation frequency $\omega$ generally is design dependent since $\omega_{i}$ changes with the design. Since it is computationally expensive to compute the term $\frac{\mathrm{d} \overline{\mathbf{q}}}{\mathrm{d} \rho_{e}}$, the value of $\boldsymbol{\lambda}$ is chosen in a way so as to eliminate $\frac{\mathrm{d} \overline{\mathbf{q}}}{\mathrm{d} \rho_{e}}$ from equation (44). This leads to the following 
condition for $\boldsymbol{\lambda}$ :

$$
2 \boldsymbol{\lambda}^{T}\left(\omega^{2} \overline{\mathbf{M}}+\omega \overline{\mathbf{C}}+\overline{\mathbf{K}}_{T}\right)+2 \overline{\mathbf{q}}^{T} \mathbf{L}=0
$$

As a result, the sensitivity of $c$ with respect to design variables $\rho_{e}$ is

$$
\frac{\mathrm{d} c}{\mathrm{~d} \rho_{e}}=2 \boldsymbol{\lambda}^{T}\left[\omega^{2} \frac{\partial \overline{\mathbf{M}}}{\partial \rho_{e}} \overline{\mathbf{q}}+\omega \frac{\partial \overline{\mathbf{C}}}{\partial \rho_{e}} \overline{\mathbf{q}}+\frac{\partial \overline{\mathbf{g}}}{\partial \rho_{e}}+(2 \omega \overline{\mathbf{M}} \overline{\mathbf{q}}+\overline{\mathbf{C}} \overline{\mathbf{q}}) \frac{\mathrm{d} \omega}{\mathrm{d} \rho_{e}}\right]
$$

and the adjoint equation is found as

$$
\left(\omega^{2} \overline{\mathbf{M}}+\omega \overline{\mathbf{C}}+\overline{\mathbf{K}}_{T}\right) \boldsymbol{\lambda}=-\mathbf{L} \overline{\mathbf{q}}
$$

In equation (46), the sensitivity of frequency $\omega$ w.r.t. the design variable $\rho_{e}$ has not yet been derived. Recalling that $\omega=\gamma \omega_{i}$ we obtain

$$
\frac{\mathrm{d} \omega}{\mathrm{d} \rho_{e}}=\frac{\gamma}{2 \omega_{i}} \frac{\mathrm{d} \omega_{i}^{2}}{\mathrm{~d} \rho_{e}}
$$

where the eigenvalue $\omega_{i}$ is found from a standard eigenvalue problem as

$$
\left(\mathbf{K}-\omega_{i}^{2} \mathbf{M}\right) \boldsymbol{\Phi}_{i}=0
$$

and the sensitivity $\frac{\mathrm{d} \omega_{i}^{2}}{\mathrm{~d} \rho_{e}}$ of a single modal eigenvalue is well known as

$$
\frac{\mathrm{d} \omega_{i}^{2}}{\mathrm{~d} \rho_{e}}=\boldsymbol{\Phi}_{i}^{T}\left[\frac{\partial \mathbf{K}}{\partial \rho_{e}}-\omega_{i}^{2} \frac{\partial \mathbf{M}}{\partial \rho_{e}}\right] \boldsymbol{\Phi}_{i}
$$

where the eigenvector is assumed to be normalized with respect to the mass matrix, i.e. $\boldsymbol{\Phi}_{i}^{T} \mathbf{M} \boldsymbol{\Phi}_{i}=1$. Otherwise, the right hand side of equation (50) should be divided by $\boldsymbol{\Phi}_{i}^{T} \mathbf{M} \boldsymbol{\Phi}_{i}$. Only the case of simple eigenvalue is considered here whereas for the case of multiple eigenvalues we refer to [43, 44, 45]. The sensitivities computed by the adjoint method have been verified by comparison with results from finite difference calculations. 
Based on the results of the nonlinear vibration analysis we compute the sensitivities and we then obtain an update of the design variables by the use of the mathematical programming software MMA [46]. This constitutes an optimization iteration and the iterative procedure is repeated until design variables and objective function converge to a prescribed tolerance.

We should point out that during the optimization procedure we keep the damping coefficient $\mu$ constant. This implies that smaller eigenvalues $\omega_{i}$ leads to a larger modal damping ratio $\xi_{i}=\mu /\left(2 \rho \omega_{i}\right)$ which might bias the optimization procedure is the damping is not sufficiently small.

\section{Optimization examples}

\subsection{Optimization of the peak of primary resonance}

As the first optimization problem we aim to minimize the peak of the primary resonance by using equation (36). The general objective function is expressed as:

$$
c\left(\rho_{e}, \omega\left(\rho_{e}\right)\right)=\overline{\mathbf{q}}^{T} \mathbf{L} \overline{\mathbf{q}}=a_{i 1}^{2}+b_{i 1}^{2}
$$

where the index $i$ denotes the degree of freedom corresponding to the lateral deflection at mid-span of the beam, $a_{i 1}$ and $b_{i 1}$ denote the corresponding coefficients of the fundamental harmonic for the lateral displacement, and $\omega$ is the frequency of the peak, which is identified using the aforementioned phase lag quadrature criterion and computed along the backbone of the primary resonance.

We bound the width by setting $b_{\min }=0.1$ and $b_{\max }=100$ and specify the volume constraint as $\alpha=0.3$ which is also set as the volume of the reference beam used as initial design for the optimization. We bound the allowable 
volume from below and since we are minimizing the amplitude for a fixed force this ensures that the volume of the final optimized design will match that of the initial reference design. Other parameters of the beam are listed in section 2.5 .

Often design optimization is performed using a linear model and for comparison we will here compare the optimized designs obtained using the nonlinear finite element model with a corresponding linear model. For the linear model we optimize for the response at the linear fundamental frequency $\omega_{1}\left(\rho_{e}\right)$ using the general formulation in equation (42) (with $\gamma=1$ ) and the sensitivities found from equations (46)-(47). The optimized results are shown in Figure 4. The design obtained by using the linear finite element model is independent of the load amplitude and it is seen to have two weak links in the structure where the design variables take their minimum value. In contrast, the optimized design obtained with the nonlinear finite element model shows a dependence on the amplitude of load. For a small amplitude of the load, $f=10 E \operatorname{Ir} / L^{3}$, the obtained design is very close to the linear design. However, when we increase the load to a larger amplitude level, $f=15 E \operatorname{Ir} / L^{3}$, the two weak links near $x=\frac{1}{4} L$ and $\frac{3}{4} L$ becomes wider. This effect is discussed in more detail in Section 5.

The linear and nonlinear frequency-amplitude curves for the two designs are shown in Figure 5. Both in linear and nonlinear analysis, the two optimized designs have a resonant peak with an amplitude significantly lower than that of the uniform design. When applying linear vibration analysis, the frequency-amplitude curves for the two optimized designs are seen to overlap. However, based on nonlinear vibration analysis we see a notable dif- 
ference in the response and the nonlinear optimized design shows a slightly smaller peak amplitude as well as a larger ratio $\omega / \omega_{1}$ for the peak of the primary resonance. A comparison of linear mode shapes of the two optimized structures normalized with respect to mass matrix is shown in Figure 5(c). While the mode shapes of two optimized structures only have slightly observable difference, they are quite different from the mode shape of the uniform design.

Thus, even with a limited design freedom that is here dictated by having only a single beam with a fixed thickness, we can see that including the nonlinearities in the optimization procedure has an influence on the nonlinear performance of the optimized designs. In the next examples we will optimize the inherent nonlinear phenomenon of superharmonic resonance.

\subsection{Optimization of superharmonic resonance}

In this case, we consider two optimization problems by using equation (42). The first one is to minimize the superharmonic resonance with a fixed load amplitude without increasing the amount of material in the beam. The second will be to maximize the superharmonic resonance also for a fixed load amplitude but without reducing the amount of material.

Considering the superharmonic resonance, the objective function is selected as

$$
c\left(\rho_{e}, \omega_{1}\left(\rho_{e}\right) / 3\right)=\overline{\mathbf{q}}^{T} \mathbf{L} \overline{\mathbf{q}}=a_{i 3}^{2}+b_{i 3}^{2}
$$

where $a_{i 3}$ and $b_{i 3}$ are the coefficients of the third harmonic terms $\cos 3 \tau$ and $\sin 3 \tau$, respectively and the subscript $i$ denotes the degree of freedom corresponding to the deflection $w$ at the mid-span of the beam. 


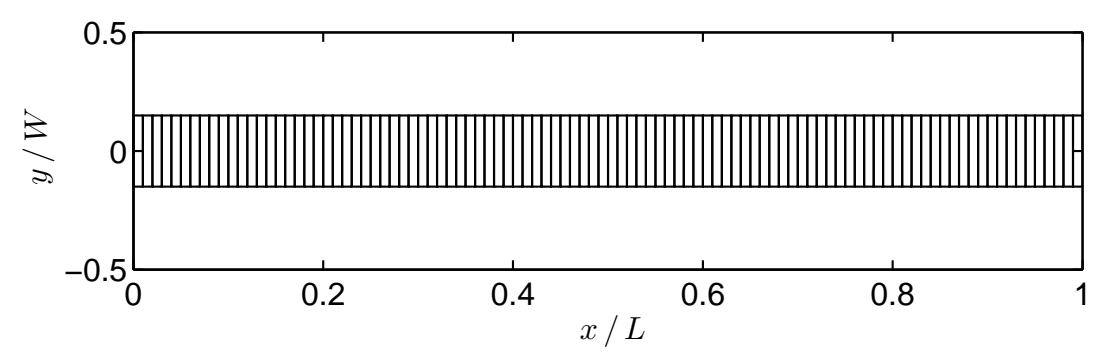

(a)

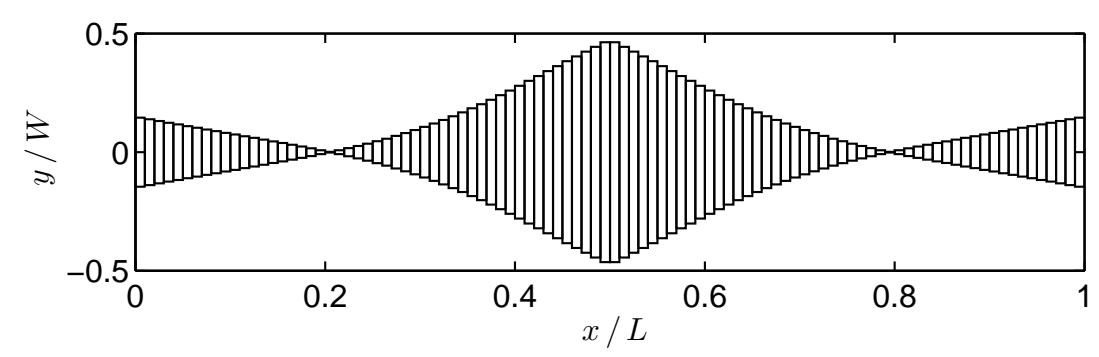

(b)

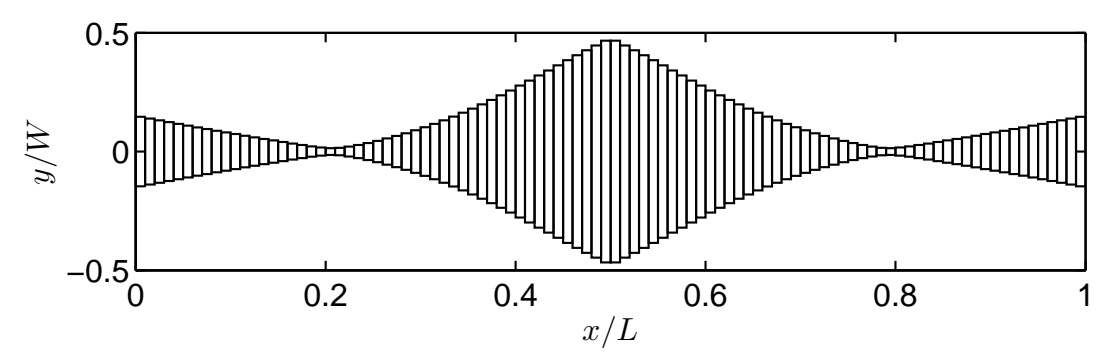

(c)

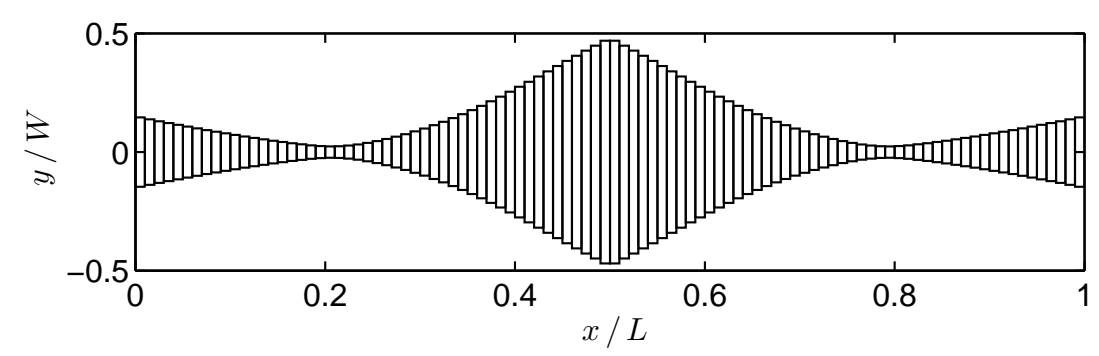

(d)

Figure 4: A doubly clamped beam with optimized width for minimizing the peak of primary resonance: (a) uniform width and initial guess; (b) optimized width using linear finite element model; (c) optimized width asing nonlinear finite element model and $\mathrm{f}=$ $60.74 \mathrm{~N}$; (d) optimized width using nonlinear finite element model and $\mathrm{f}=91.11 \mathrm{~N}$. 


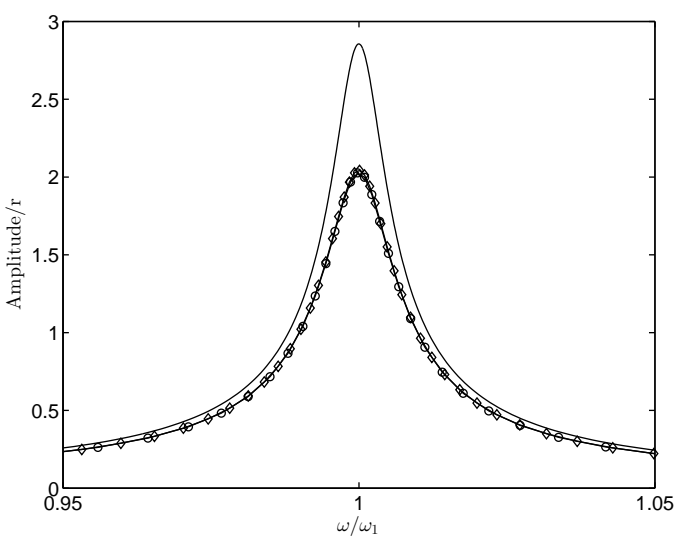

(a)

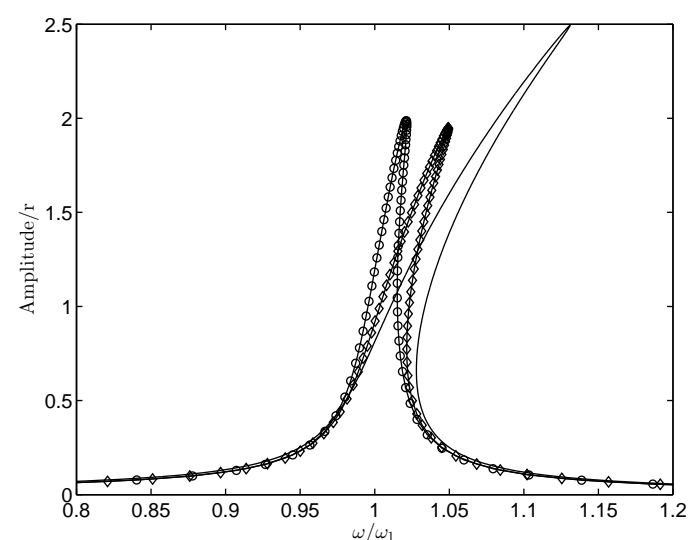

(b)

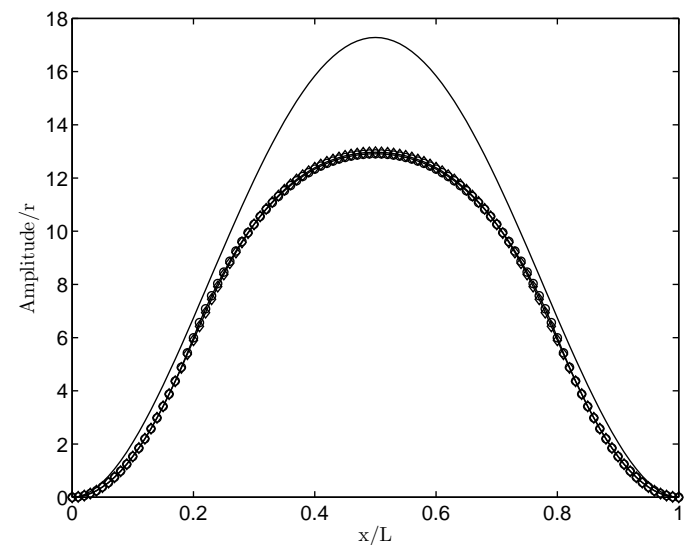

(c)

Figure 5: A doubly clamped beam with 2 ptimized width for minimizing the peak of primary resonance: '.' uniform width; 'o' optimized width using linear finite element model; ' $\diamond$ ' optimized width using nonlinear finite element model and $\mathrm{f}=91.11 \mathrm{~N}$ : (a) linear analysis; (b) nonlinear analysis; (c) linear modal analysis. 
The optimization problem is then changed to

$$
\begin{gathered}
\underset{\rho_{e}}{\min / \max } \quad c=a_{i 3}^{2}+b_{i 3}^{2} \\
\text { s.t. }: \quad \omega=\frac{1}{3} \omega_{1}
\end{gathered}
$$

subjected also to the additional constraints from equation (36) (except $b_{i 1}=$ 0 ) using either a minimum or a maximum volume fraction. Here, $\omega_{1}$ is the $1^{\text {st }}$ eigenvalue so that $\omega=\frac{1}{3} \omega_{1}$ corresponds to a frequency close to the first superharmonic resonance. The allowable volume is $\alpha=0.3$. The initial structure is a beam with uniform width and a bottom limit of the width set to $b_{\min }=10$.

In the case of an external load given by $607.4 \mathrm{~N}$ we obtain the optimized structure shown in Figure 6. The frequency-amplitude curves for the uniform-width design and the optimized design are compared in Figure 9. It can be seen that the superharmonic resonance is reduced significantly with the optimized width distribution even though the total volume is unchanged. The effect of modifying the design can clearly be seen by viewing the structural response in the time domain. Figure 10 shows the periodic response in the time domain for the peak of the frequency-amplitude curve in Figure 9. The design with uniform width distribution produces a distorted signal with higher harmonics whereas the design with optimized width reduces the higher-order harmonics effectively.

Next we aim to maximize the superharmonic resonant response. We pose this as a minimization problem with $1 / c$ as the objective. The volume constraint is set as $\alpha=0.3$ but we now bound the volume from above. The initial design, bottom limit of the width and the external load are the same as for the minimization problem solved above. The optimized structure is 


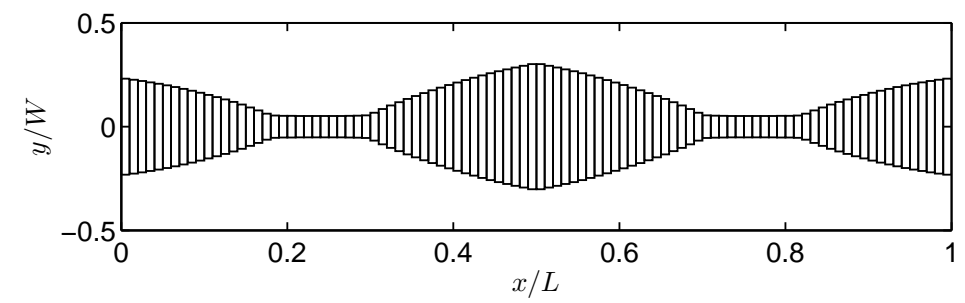

Figure 6: A doubly clamped beam with optimized width for minimizing superharmonic resonance around $\omega_{1} / 3$

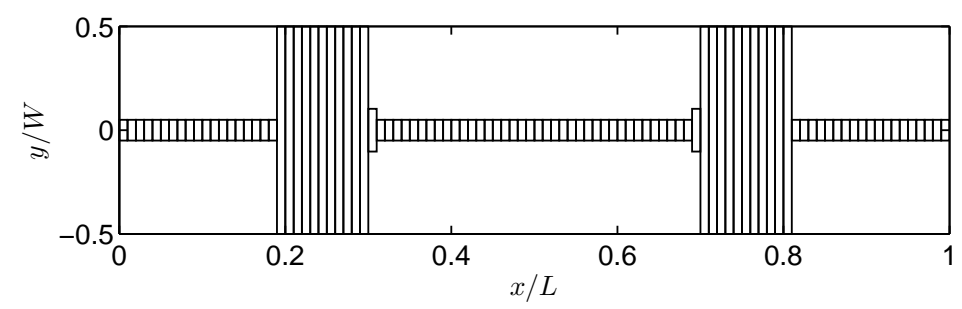

Figure 7: A doubly clamped beam with optimized width for maximizing superharmonic resonance around $\omega_{1} / 3$

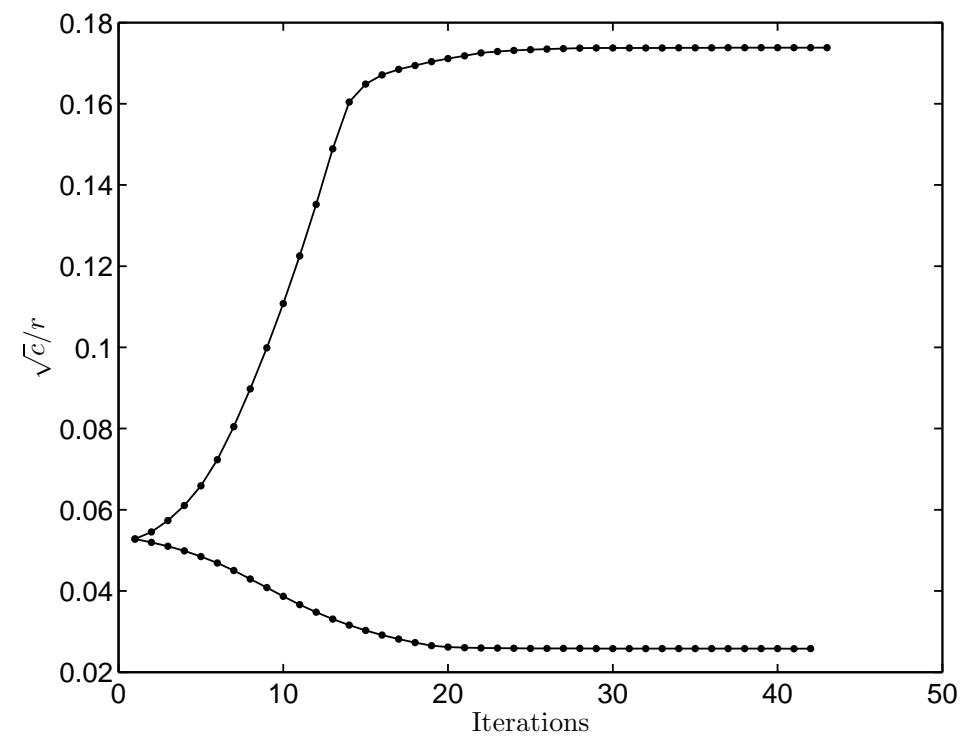

Figure 8: Iteration history of the objective during optimization. 


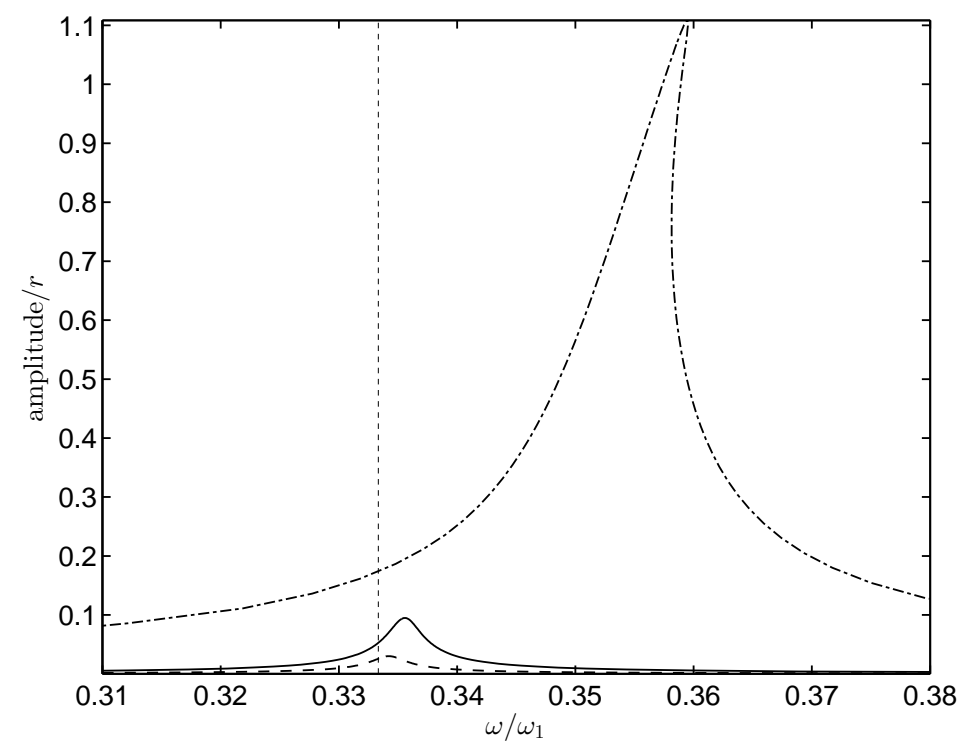

Figure 9: Frequency-amplitude curves of superharmonic resonance around $\omega_{1} / 3$ : solid line: uniform; dashed line: minimized superharmonic resonance; dot dashed line: maximized superharmonic resonance; dotted line: $\frac{\omega}{\omega_{1}}=\frac{1}{3}$. 


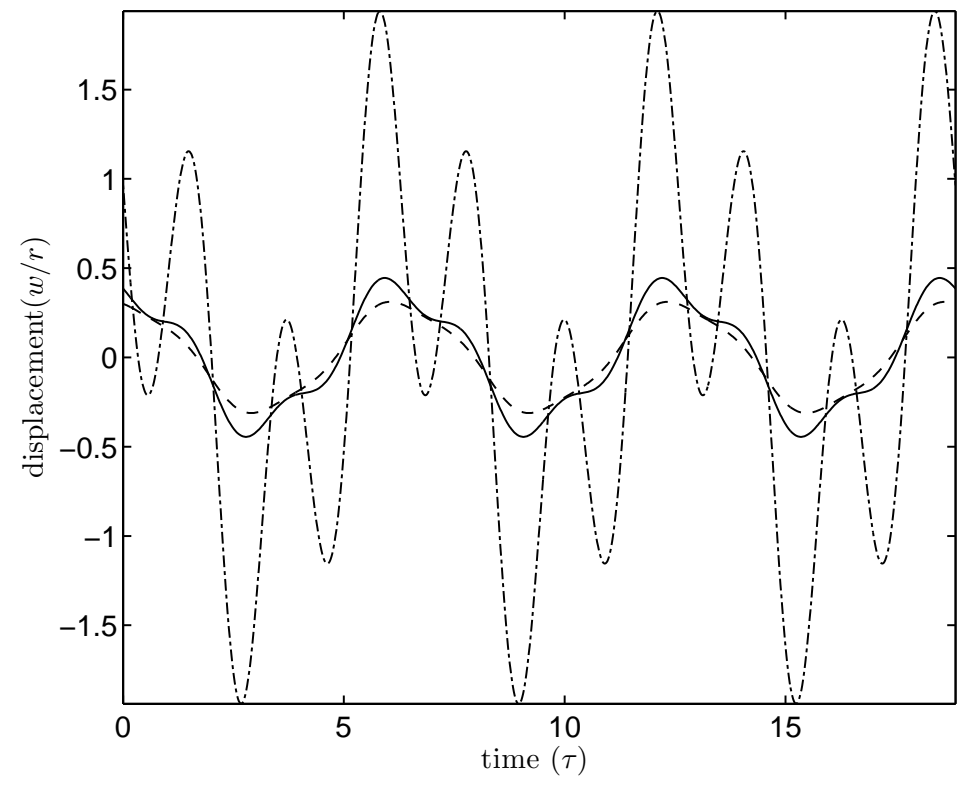

Figure 10: The responses for the peak of superharmonic resonance around $\omega_{1} / 3$ : solid line: uniform; dashed line: minimized superharmonic resonance; dot dashed line: maximized superharmonic resonance. 
shown in Figure 7 and the evolution of the objective during the optimization iterations for both optimization problems is shown in Figure 8. The frequency-amplitude curves for uniform and optimized designs are shown in Figure 9. The time-domain response that corresponds to the peak of the frequency-amplitude curve is shown in Figure 10 and we see here that the response at the mid-span of the beam is dominated by the third-order harmonic for this optimized design.

\section{Discussion}

Numerical experience shows that it is important to select necessary and sufficient harmonics in the Fourier expansion. A general guideline about completeness and balance ability was given by Leung and Fung [19]. For the sake of completeness, the second and third order harmonics are essential for the case of quadratic and cubic nonlinearities. Particularly, the second order harmonic is necessary for describing the longitudinal motion of stretching. For the sake of balance ability, all harmonics up to and including the sixth order are included in the Fourier expansion here. Additionally it should be mentioned that the number of elements should be sufficient to predict the modes around the highest frequency in the Fourier expansion.

It is noted that when we minimize the resonant peak, the optimized design based on the nonlinear finite element model has a larger width around $x=\frac{1}{4} L$ and $x=\frac{3}{4} L$, whereas the cross section area vanishes in the optimized design based on the linear finite element model. There is a simple physical interpretation for this phenomenon. Based on the theory of linear vibration, the cross section area would be allowed to vanish at inner points where the 
material does not contribute to the bending strain energy. In contrast, the bending and stretching deformations are coupled in the nonlinear case. In our example, the bending deformation causes stretching strain energy due to midplane stretching. As shown in Figure 11, where $\phi_{1}$ denotes the deformation $w$ for the first linear mode of a doubly clamped beam, the bending strain energy is proportional to $\left(\frac{\partial^{2} \phi_{1}}{\partial x^{2}}\right)^{2}$ and the strain energy associated with midplane stretching is proportional to $\left(\frac{\partial \phi_{1}}{\partial x}\right)^{4}$. The strongest midplane stretching effect occurs where $\left(\frac{\partial \phi_{1}}{\partial x}\right)^{4}$ reaches the local maximum and $\left(\frac{\partial^{2} \phi_{1}}{\partial x^{2}}\right)^{2}$ reaches the local minimum. So as the load increases, the coupling effect will lead to an increased width around $x=\frac{1}{4} L$ and $x=\frac{3}{4} L$.

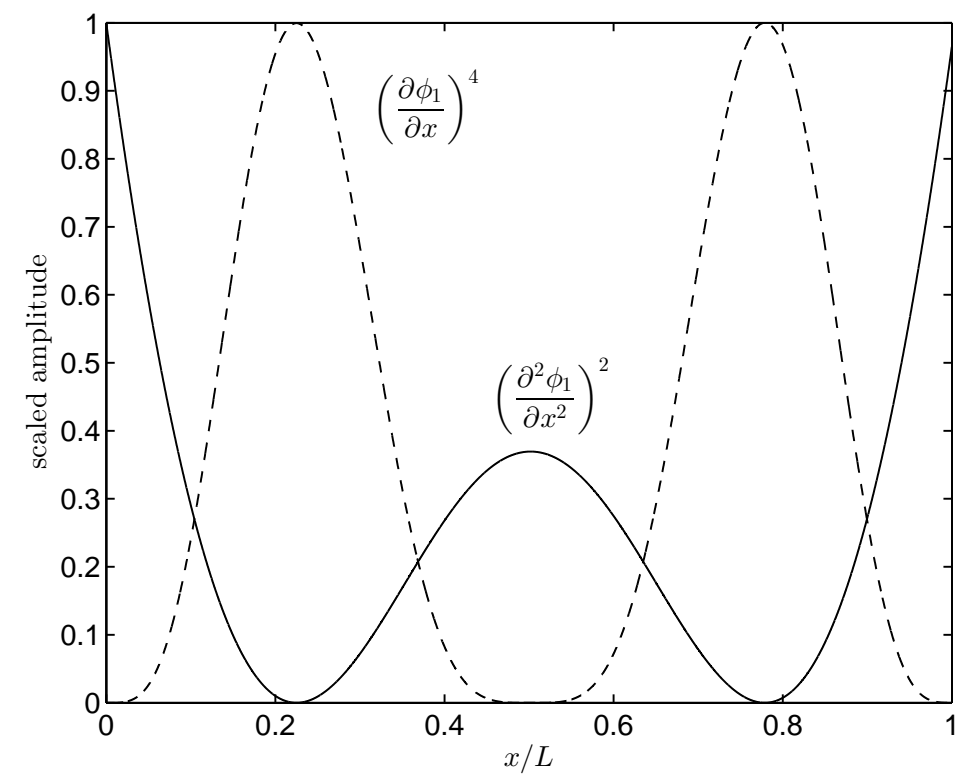

Figure 11: Strain energy distribution for the first linear mode. 


\section{Conclusion}

A methodology here is proposed for optimizing the nonlinear forced response of geometrically nonlinear beam structures with quadratic and cubic nonlinearities. The nonlinear vibration analysis is performed via the combination of the finite element method and the incremental harmonic balance method. Design sensitivities are derived from the nonlinear response using an adjoint approach and we solve the optimization problem using a gradientbased approach with the mathematical programming tool MMA. We include examples that demonstrate the effectiveness of the proposed methodology. By minimizing the amplitude of the primary resonance we demonstrate the effect of including the nonlinearity in the optimization procedure, although the difference in the final response is not very large. However, it is noted that the optimization based on nonlinear finite element model leads to a more robust design without the weak links that are found in the linear design. In the case of optimizing the superharmonic resonance, it is necessary to employ the nonlinear finite element model. In this case we see a significant effect on the structural response when we either minimize or maximize the contribution from the third-order harmonic.

This methodology has shown a promising ability in optimizing the design of the nonlinear dynamic response of beam structures and may find applications for vibration-based energy harvesting and MEMS structures by taking into account their nonlinear performance. The work can be extended to general finite element models by using the alternating frequency/time domain method. Future work includes optimization of frame structures and 2D continuum structure based on geometrically nonlinear finite element analysis. 


\section{Acknowledgements}

This project was supported by ERC Starting Grant 279529 INNODYN. The authors also thank Krister Svanberg in Royal Institute of Technology (KTH), Sweden, for the permission to use MMA and Ole Sigmund for valuable comments and suggestions.

\section{References}

[1] J. F. Rhoads, S. W. Shaw, K. L. Turner, Nonlinear dynamics and its applications in micro- and nanoresonators, Journal of Dynamic Systems, Measurement, and Control 132 (2010) 034001.

[2] J. J. Thomsen, Vibrations and Stability, Order and Chaos, McGRAWHILL, 1997.

[3] W. Lacarbonara, H. Yabuno, Refined models of elastic beams undergoing large in-plane motions: Theory and experiment, International Journal of Solids and Structures 43 (2006) 5066 - 5084 .

[4] K. Wojciechowski, R. Olsson, M. Baker, J. Wittwer, Low vibration sensitivity mems resonators (2007) $1220-1224$.

[5] R. Mestrom, R. Fey, K. Phan, H. Nijmeijer, Simulations and experiments of hardening and softening resonances in a clamped-clamped beam mems resonator, Sensors and Actuators A: Physical 162 (2010) $225-234$.

[6] A. Hajati, S.-G. Kim, Ultra-wide bandwidth piezoelectric energy harvesting, Applied Physics Letters 99 (2011) 083105. 
[7] S. L. Lau, Y. K. Cheung, Amplitude incremental variational principle for nonlinear vibration of elastic systems, Journal of Applied Mechanics 48 (1981) 959-964.

[8] S. H. Chen, Y. K. Cheung, H. X. Xing, Nonlinear vibration of plane structures by finite element and incremental harmonic balance method, Nonlinear Dynamics 26 (2001) 87-104.

[9] R. Lewandowski, Non-linear, steady-state vibration of structures by harmonic balance/finite element method, Computers \& Structures 44 (1992) 287 - 296. Special Issue: WCCM II.

[10] R. Lewandowski, Computational formulation for periodic vibration of geometrically nonlinear structurespart 1: Theoretical background, International Journal of Solids and Structures 34 (1997) 1925 - 1947.

[11] R. Lewandowski, Computational formulation for periodic vibration of geometrically nonlinear structurespart 2: Numerical strategy and examples, International Journal of Solids and Structures 34 (1997) 1949 1964.

[12] T. Cameron, J. Griffin, An alternating frequency/time domain method for calculating the steady-state response of nonlinear dynamic systems, Journal of Applied Mechanics 56 (1989) 149-154.

[13] R. C. Maple, P. I. King, P. D. Orkwis, J. M. Wolff, Adaptive harmonic balance method for nonlinear time-periodic flows, Journal of Computational Physics 193 (2004) 620 - 641. 
[14] Y. Cheung, S. Chen, S. Lau, Application of the incremental harmonic balance method to cubic non-linearity systems, Journal of Sound and Vibration 140 (1990) $273-286$.

[15] A. Leung, S. Chui, Non-linear vibration of coupled duffing oscillators by an improved incremental harmonic balance method, Journal of Sound and Vibration 181 (1995) $619-633$.

[16] A. Raghothama, S. Narayanan, Bifurcation and chaos in geared rotor bearing system by incremental harmonic balance method, Journal of Sound and Vibration 226 (1999) $469-492$.

[17] J. Didier, J.-J. Sinou, B. Faverjon, Nonlinear vibrations of a mechanical system with non-regular nonlinearities and uncertainties, Communications in Nonlinear Science and Numerical Simulation 18 (2013) 3250 3270 .

[18] A. Y. T. Leung, T. C. Fung, Phase increment analysis of damped duffing oscillators, International Journal for Numerical Methods in Engineering 28 (1989) 193-209.

[19] A. Y. T. Leung, T. C. Fung, Non-linear steady state vibration of frames by finite element method, International Journal for Numerical Methods in Engineering 28 (1989) 1599-1618.

[20] B. Cochelin, C. Vergez, A high order purely frequency-based harmonic balance formulation for continuation of periodic solutions, Journal of Sound and Vibration 324 (2009) $243-262$. 
[21] J. Thomas, E. Dowell, K. Hall, Nonlinear inviscid aerodynamic effects on transonic divergence, flutter, and limit-cycle oscillations, AIAA Journal 40 (2002) 638-646.

[22] K. Hall, J. Thomas, W. Clark, Computation of unsteady nonlinear flows in cascades using a harmonic balance technique, AIAA Journal 40 (2002) 879-886.

[23] A. LaBryer, P. Attar, A harmonic balance approach for large-scale problems in nonlinear structural dynamics, Computers \& Structures 88 (2010) $1002-1014$.

[24] M. Thothadri, F. C. Moon, Nonlinear system identification of systems with periodic limit-cycle response, Nonlinear Dynamics 39 (2005) 63-77.

[25] H. Ahmadian, H. Jalali, Generic element formulation for modelling bolted lap joints, Mechanical Systems and Signal Processing 21 (2007) $2318-2334$.

[26] N. Coudeyras, J.-J. Sinou, S. Nacivet, A new treatment for predicting the self-excited vibrations of nonlinear systems with frictional interfaces: The constrained harmonic balance method, with application to disc brake squeal, Journal of Sound and Vibration 319 (2009) 1175 1199.

[27] H. Liao, W. Sun, A new method for predicting the maximum vibration amplitude of periodic solution of non-linear system, Nonlinear Dynamics (2012) 1-14. 
[28] N. Olhoff, Optimization of vibrating beams with respect to higher order natural frequencies, Journal of Structural Mechanics 4 (1976) 87-122.

[29] N. Olhoff, R. Parbery, Designing vibrating beams and rotating shafts for maximum difference between adjacent natural frequencies, International Journal of Solids and Structures 20 (1984) $63-75$.

[30] M. P. Bendsøe, N. Olhoff, A method of design against vibration resonance of beams and shafts, Optimal Control Applications and Methods 6 (1985) 191-200.

[31] R. Meske, B. Lauber, E. Schnack, A new optimality criteria method for shape optimization of natural frequency problems, Structural and Multidisciplinary Optimization 31 (2006) 295-310.

[32] N. Olhoff, B. Niu, G. Cheng, Optimum design of band-gap beam structures, International Journal of Solids and Structures 49 (2012) 3158 3169.

[33] S. Paquin, Y. St-Amant, Improving the performance of a piezoelectric energy harvester using a variable thickness beam, Smart Materials and Structures 19 (2010) 105020.

[34] J. M. Dietl, E. Garcia, Beam shape optimization for power harvesting, Journal of Intelligent Material Systems and Structures 21 (2010) 633646.

[35] B. Stanford, P. Beran, M. Kurdi, Adjoint sensitivities of time-periodic nonlinear structural dynamics via model reduction, Computers and Structures 88 (2010) 1110 - 1123. 
[36] B. Stanford, P. Beran, R. Snyder, M. Patil, Stability and power optimality in time-periodic flapping wing structures, Journal of Fluids and Structures 38 (2013) $238-254$.

[37] M. P. Bendsøe, O. Sigmund, Topology Optimization: Theory, Methods and Applications, Springer, 2003.

[38] M. Crisfield, A fast incremental/iterative solution procedure that handles snap-through, Computers \& Structures 13 (1981) 55 - 62.

[39] M. Peeters, R. Vigui, G. Srandour, G. Kerschen, J. C. Golinval, Nonlinear normal modes, part ii: Toward a practical computation using numerical continuation techniques, Mechanical Systems and Signal Processing 23 (2009) $195-216$.

[40] E. P. Petrov, Advanced analysis and optimization of nonlinear resonance vibrations in gas-turbine structures with friction and gaps, IUTAM SYMPOSIUM ON EMERGING TRENDS IN ROTOR DYNAMICS 25 (2011) 297-307.

[41] M. Peeters, G. Kerschen, J. Golinval, Modal testing of nonlinear vibrating structures based on nonlinear normal modes: Experimental demonstration, Mechanical Systems and Signal Processing 25 (2011) 1227 1247.

[42] M. Peeters, G. Kerschen, J. Golinval, Dynamic testing of nonlinear vibrating structures using nonlinear normal modes, Journal of Sound and Vibration 330 (2011) 486 - 509. 
[43] A. P. Seyranian, Sensitivity analysis of multiple eigenvalues, Journal of Structural Mechanics 21 (1993) 261-284.

[44] A. Seyranian, E. Lund, N. Olhoff, Multiple eigenvalues in structural optimization problems, Structural optimization 8 (1994) 207-227.

[45] J. Du, N. Olhoff, Topological design of freely vibrating continuum structures for maximum values of simple and multiple eigenfrequencies and frequency gaps, Structural and Multidisciplinary Optimization 34 (2007) 91-110.

[46] K. Svanberg, The method of moving asymptotes - a new method for structural optimization, International Journal for Numerical Methods in Engineering 24 (1987) 359-373. 